\title{
Epigenetic reprogramming to prevent genetic cardiomyopathy
}

\author{
Jamie R. Johnston, Daniel F. Selgrade, and Elizabeth M. McNally \\ Center for Genetic Medicine, Northwestern University Feinberg School of Medicine, Chicago, Illinois, USA.
}

\begin{abstract}
Mutations in the gene that codes for lamin A/C (LMNA) are a common cause of adult-onset cardiomyopathy and heart failure. In this issue of the $J C l$, Guenantin and Jebeniani et al. identify impaired cardiomyocyte development and maturation as a prenatal feature in a model of laminopathy. Cardiomyocytes carrying the Lmna point mutation $\mathrm{H} 222 \mathrm{P}$ misexpressed genes involved in the epithelial-mesenchymal transition and showed decreased methylation at the fourth lysine of histone H3 (H3K4). Notably, inhibiting lysine-specific demethylase 1 in the LMNA H222P mouse model treated this congenital form of cardiomyopathy and improved survival in utero. These data highlight early epigenomic modifications in lamin A/C-mediated pathology and indicate a unique therapeutic strategy for cardiomyopathy.
\end{abstract}

\section{LMNA mutations}

Mutations in the gene encoding A-type lamins (LMNA) cause a broad spectrum of human diseases, collectively referred to as laminopathies. Dominant LMNA mutations most commonly cause cardiomyopathy with irregular heart rhythms, but LMNA mutations also cause skeletal myopathies, lipodystrophies, and premature aging syndromes (1-4). The LMNA gene is expressed in virtually all differentiated somatic cells and encodes two predominant alternatively spliced isoforms, lamins A and C (lamin A/C) (5). Lamin $\mathrm{A} / \mathrm{C}$ are intermediate filament proteins that, along with B-type lamins, are major constituents of the inner nuclear envelope and form the nuclear lamina (6). In addition to providing a structural scaffolding for the nucleus, the nuclear lamina participates in mechanotransduction, chromatin organization, regulation of gene transcription, and DNA repair/replication (6). Despite the near-ubiquitous expression of LMNA, laminopathies predominantly affect mechanically active tissues such heart and skeletal muscle (7).

LMNA mutations are responsible for $5 \%$ to $8 \%$ of genetic dilated cardiomyopathy (DCM) (8). The average age of disease onset in LMNA mutation carriers is variable, and symptoms generally occur in the third decade of life. Strikingly, it has been reported that the penetrance of LMNA mutations approaches $100 \%$ by 60 years of age, and affected individuals often require cardiac transplantation because of end-stage heart failure (9). Compared with other cardiomyopathy genes, LMNA mutations are associated with a high incidence of sudden cardiac death due to conduction defects and atrial/ventricular arrhythmias, manifestations that often precede the development of overt DCM (9).

Many LMNA missense mutations are thought to act through a dominant-negative mechanism. There are two prevailing theories on the molecular basis

\section{Related Article: https://doi.org/10.1172/JCI136488}

of LMNA-related DCM (6). A structural hypothesis proposes that the physical integrity of cardiac nuclei is compromised, leading to increased cell death and a progressive decline in myocardial contractile performance. The gene regulation hypothesis suggests that chromatin organization and interactions among tissue-specific transcription/epigenetic factors are perturbed, resulting in aberrant gene expression and activation of pathological signaling pathways. There is also compelling evidence implicating disrupted cardiomyocyte nuclear mechanics and mechanotransduction in LMNA-related $\operatorname{DCM}(10,11)$. These pathogenic modes are likely not mutually exclusive, but rather, they might cooperate through a complex interaction, ultimately culminating in a final clinical phenotype.

\section{Cardiac defects originate during development}

In this issue of the JCI, Guénantin and Jebeniani et al. use the Lmna mouse model engineered with the missense $\mathrm{H} 222 \mathrm{P}$ mutation to link lamin $\mathrm{A} / \mathrm{C}$ with early developmental cardiomyopathy (12). The H222P mutation was originally described in a small, three-generation family with autosomal dominant Emery Dreifuss muscular dystrophy; two of the affected three family members had arrhythmias (13). As one of the first knockin models for laminopathy, mice were generated to carry this variant, which is positioned within the rod domain and a linker region (14). Lmna ${ }^{\mathrm{H} 222 \mathrm{P} / \mathrm{H} 222 \mathrm{P}}$ mice develop locomotion defects and ventricular dilation, resulting in early mortality between 9 and 13 months (14).

Guénantin, Jebeniani, and colleagues now report that cardiac defects in $L m n a^{\mathrm{H} 222 \mathrm{P} / \mathrm{H} 222 \mathrm{P}}$ originate during development, as early as embryonic day 13.5, and associate with a distinct transcriptional and morphological profile (12).

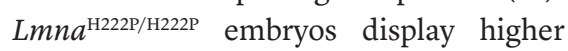
rates of lethality compared with normal or heterozygous controls. To more carefully 


\section{A Normal development}

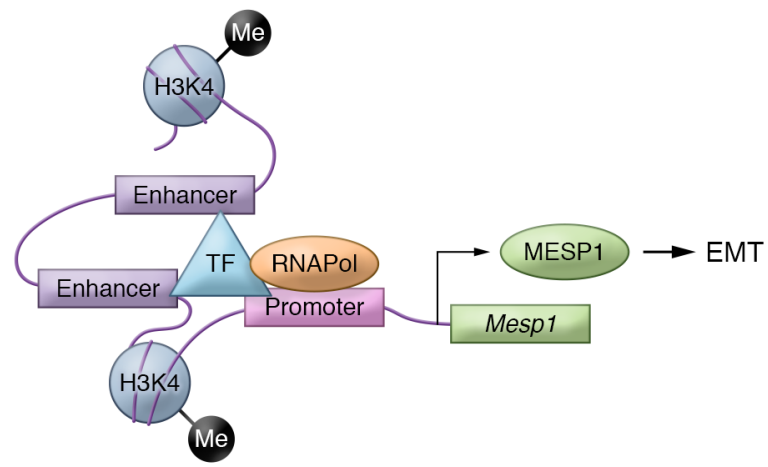

B Lmna $a^{\mathrm{H} 222 \mathrm{P} /+}$ ES-derived cardiomyocytes

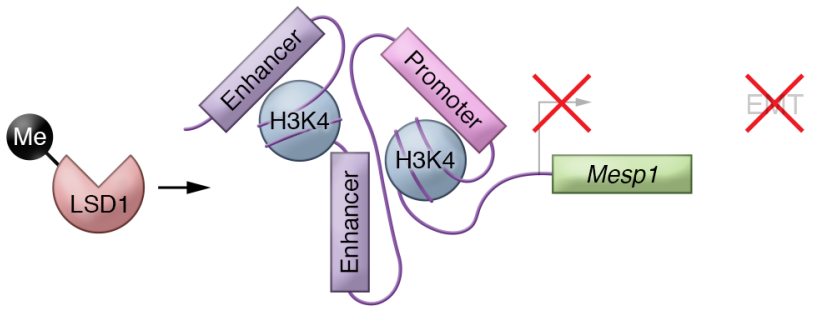

C Lmna ${ }^{\text {H222P/H222P }}$ embryonic heart + LSD1 inhibitor

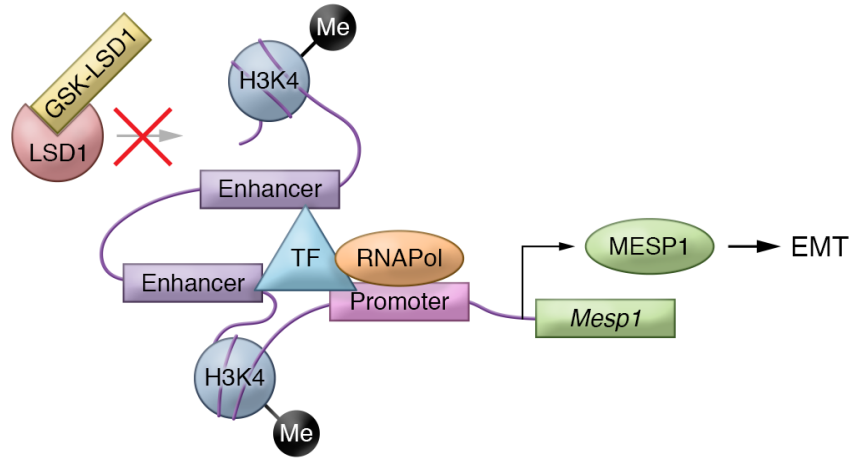

Figure 1. The H222P Lmna mutation inhibits proper EMT in developing cardiomyocytes. (A) In the normal developing heart, MESP1 is required for normal EMT. (B) In Lmna ${ }^{\mathrm{H} 222 \mathrm{P} /+}$ ESC-derived cardiomyocytes, activity of histone demethylase LSD1 results in decreased MESP1, resulting in reduced EMT. (C) Inhibition of LSD1 via a small molecule GSK1-LSD restores cardiac function in Lmna ${ }^{\text {H222P/H222P }}$ mice. Restoration of proper chromatin accessibility represents an attractive therapeutic target to prevent cardiomyopathy in laminopathies. TF, transcription factor; RNAPol, RNA polymerase.

study cardiomyocyte differentiation and specification, the authors studied heterozygous Lmna ${ }^{\mathrm{H} 222 \mathrm{P} /+}$ mouse embryonic stem cell (ESC) lines. The authors identified lysine-specific demethylase 1 (LSD1, also known as lysine (K)-specific demethylase $1 \mathrm{~A}, \mathrm{KDM} 1 \mathrm{~A}$ ) as a central regulator of cardiac development through its influence on the mesoderm posterior 1 (Mesp1) gene (12). Mesp1 encodes mesoderm posterior 1, a basic helix-loop-helix transcription factor that regulates epithelial-mesenchymal transition (EMT) and cell specification
(15). The Mesp1 gene promoter was heavily methylated in $\mathrm{Lmna}^{\mathrm{H} 222 \mathrm{P} /+}$ ESC lines compared with WT controls (Figure 1). Downregulation of the $L s d 1$ gene was sufficient to rescue the defective epigenetic landscape in mesodermal cells, improving cardiac contractility. Finally, a small molecule inhibitor of LSD1 activity also rescued the lethality of $L_{m} a^{\mathrm{H} 222 \mathrm{P} / \mathrm{H} 222 \mathrm{P}}$ embryos, suggesting epigenomic modification of genes critical to EMT is important in laminopathy (12).

The observations made in Lmna-H222P heterozygous ESCs imply a congenital ori- gin for lamin A/C-related cardiomyopathy. Although present from conception, cardiomyopathic genetic mutations manifest later in life with an age-dependent onset (9). The findings from Guénantin, Jebeniani, and colleagues suggest some of these mutations may have early, even developmental, components (12). Inherited cardiomyopathic LMNA mutations manifest with conduction system disease often as the first finding but only after being present for decades. Ascertaining whether some of these same pathways for EMT are disrupted in mature human myocardium and conduction systems will be a critical next step. Whether other LMNA mutations, missense or truncating, act through these same pathways is also not known. Carriers of truncating LMNA mutations are at increased risk for more severe arrhythmia phenotypes; therefore, EMT or MESP1 and/or its targets may be especially relevant for the conduction system.

\section{Conclusions and clinical implications}

The chromatin changes observed may also indicate the presence of a generally important pathway outside of LMNA. The LSD1 inhibitor improved contractility of normal embryoid bodies compared with untreated embryoid bodies. Enhanced contractility was also observed in vivo at postnatal days one and three, hinting at LMNA-independent effects of the compound. LSD1 is ubiquitously expressed and, therefore, targeting LSD1 should alter more than cardiomyocyte function. Human patients with H222P, just like the mouse model, have accompanying musculoskeletal defects that include tendon contractures and impaired muscle growth $(13,14)$. LSD1 inhibition may have efficacy in promoting skeletal muscle development and maturation $(16,17)$, and these known roles could influence its use.

Further studies are needed to ascertain whether the LSD1 inhibitor affects the adult heart and especially the cardiac electrical system, as it does embryonic hearts, and this could be especially relevant to disease given the onset and findings in human laminopathy. Notably, when the pregnant female mice were treated with the GSK-LSD1 inhibitor, myocardial contraction in the pregnant dams may have improved, which itself could have bearing on pregnancy outcomes. This observation raises the possibility that, in addition to targeting histones in the nucle- 
us, LSD1 targets the contractile apparatus in cardiomyocytes, as LSD1 is known to also demethylate non-histone proteins (18). Contractile effects would have obvious therapeutic implications for heart failure and perhaps irrespective of etiology.

LSD1 also demonstrates notable promiscuity in downstream targets involved in cellular response to neuroendocrine and metabolic signals, both of which play a role in the coordination of cardiac development $(15,19)$. Furthermore, recent studies suggest LMNA regulates chromatin status via polycomb protein (20), supporting a direct downstream alternative pathway to explain the change in chromatin state. Nevertheless, the inhibition of the LSD1 pathway demonstrates improved cardiac outcomes in the homozygous mouse model, suggesting a new therapeutic target for laminopathies.

\section{Acknowledgments}

The authors received research funding from the NIH.

Address correspondence to: Elizabeth M. McNally, 303 E. Superior St., Simpson Querrey 5-516, Center for Genetic Medicine, Northwestern University Feinberg School of Medicine, Chicago, Illinois 60611, USA. Phone: 312.503.5600; Email: elizabeth.mcnally@northwestern.edu.
1. Fatkin D, et al. Missense mutations in the rod domain of the lamin $\mathrm{A} / \mathrm{C}$ gene as causes of dilated cardiomyopathy and conduction-system disease. NEngl J Med. 1999;341(23):1715-1724.

2. Raffaele Di Barletta M, et al. Different mutations in the LMNA gene cause autosomal dominant and autosomal recessive Emery-Dreifuss muscular dystrophy. Am J Hum Genet. 2000;66(4):1407-1412.

3. MacLeod HM, Culley MR, Huber JM, McNally EM. Lamin A/C truncation in dilated cardiomyopathy with conduction disease. BMC Med Genet. 2003;4:4.

4. Bonne G, et al. Mutations in the gene encoding lamin A/C cause autosomal dominant Emery-Dreifuss muscular dystrophy. Nat Genet. 1999;21(3):285-288.

5. Lin F, Worman HJ. Structural organization of the human gene encoding nuclear lamin A and nuclear lamin C. JBiol Chem. 1993;268(22):16321-16326.

6. Ho CY, Lammerding J. Lamins at a glance. J Cell Sci. 2012;125(pt 9):2087-2093.

7. Kirby TJ, Lammerding J. Emerging views of the nucleus as a cellular mechanosensor. Nat Cell Biol. 2018;20(4):373-381.

8. McNally EM, Mestroni L. Dilated cardiomyopathy: genetic determinants and mechanisms. Circ Res. 2017;121(7):731-748.

9. Hasselberg NE, et al. Lamin A/C cardiomyopathy: young onset, high penetrance, and frequent need for heart transplantation. Eur Heart $J$. 2018;39(10):853-860.

10. Nikolova V, et al. Defects in nuclear structure and function promote dilated cardiomyopathy in lamin A/C-deficient mice. JClin Invest. 2004;113(3):357-369.

11. Lammerding J, et al. Lamin A/C deficiency causes defective nuclear mechanics and mechano- transduction. J Clin Invest. 2004;113(3):370-378.

12. Guénantin AC, et al. Targeting the histone demethylase LSD1 prevents cardiomyopathy in a mouse model of laminopathy. JClin Invest. 2020;131(1):e136488.

13. Bonne $\mathrm{G}$, et al. Clinical and molecular genetic spectrum of autosomal dominant Emery-Dreifuss muscular dystrophy due to mutations of the lamin A/C gene. Ann Neurol. 2000;48(2):170-180.

14. Arimura T, et al. Mouse model carrying H222P-Lmna mutation develops muscular dystrophy and dilated cardiomyopathy similar to human striated muscle laminopathies. Hum Mol Genet. 2005;14(1):155-169.

15. Chan SS, et al. Mesp1 patterns mesoderm into cardiac, hematopoietic, or skeletal myogenic progenitors in a context-dependent manner. Cell Stem Cell. 2013;12(5):587-601.

16. Choi J, Jang H, Kim H, Kim ST, Cho EJ, Youn HD. Histone demethylase LSD1 is required to induce skeletal muscle differentiation by regulating myogenic factors. Biochem Biophys Res Commun. 2010;401(3):327-332.

17. Choi J, et al. Modulation of lysine methylation in myocyte enhancer factor 2 during skeletal muscle cell differentiation. Nucleic Acids Res. 2014;42(1):224-234.

18. Nicholson TB, Chen T. LSD1 demethylates histone and non-histone proteins. Epigenetics. 2009;4(3):129-132.

19. Anan K, et al. LSD1 mediates metabolic reprogramming by glucocorticoids during myogenic differentiation. Nucleic Acids Res. 2018;46(11):5441-5454.

20. Bianchi A, et al. Dysfunctional polycomb transcriptional repression contributes to lamin A/C-dependent muscular dystrophy. J Clin Invest. 2020;130(5):2408-2421. 